\title{
A novel approach for the separation of neutral analytes by means of electrochemically assisted injection coupled to capillary electrophoresis-mass spectrometry
}

\author{
Rebekka Scholz and Frank-Michael Matysik* \\ Received 12th October 2010, Accepted 31st January 2011 \\ DOI: 10.1039/c0an00794c
}

\begin{abstract}
A novel concept for capillary electrophoresis-mass spectrometry (CE-MS) studies of neutral analytes is presented. The experimental approach is based on the implementation of electrochemically assisted injection (EAI) which enables the generation of charged species from neutral analytes. In this way electrophoretic separations can be performed without the use of surfactants leading to an excellent compatibility with ESI-MS. Various ferrocene species have been used as model compounds to demonstrate the potential of the EAI-CE-MS technique.
\end{abstract}

Capillary electrophoresis (CE) is a powerful high-efficiency separation technique with a wide range of applications. ${ }^{1}$ The coupling of CE with electrospray ionization-mass spectrometry (ESI-MS) is very attractive due to the excellent selectivity and sensitivity of MS detection. One challenging problem is the separation of neutral analytes by CE in combination with ESI-MS. In general, CE separations of neutral analytes can be realized by means of micellar electrokinetic capillary chromatography (MECC) introduced by Terabe. $^{2}$ However, the use of a relatively high concentration of surfactants such as sodium dodecyl sulfate results in a significantly reduced compatibility with ESI-MS. This is due to influence of the surfactants on the ESI processes affecting the ionization efficiency of the analytes. ${ }^{3}$ In recent years some efforts have been undertaken to reduce these limitations. ${ }^{4,5}$

This communication presents the concept of electrochemically assisted injection (EAI) in combination with CE-MS as a novel means for the determination of neutral analytes by CE-MS. The approach of EAI-CE-MS is based on the electrochemical conversion of redox-active analytes during the injection step into the capillary flow system. The motivation for this methodological development involves several aspects. Firstly, EAI offers the possibility of generating ionic species in terms of electrochemical conversion of neutral analytes which allows CE separation based on the different electrophoretic mobilities of the charged species. Secondly, mechanistic studies of electrochemical reactions can be performed taking advantage from the powerful CE-MS technique for product

Institute of Analytical Chemistry, Chemo- and Biosensors, University of Regensburg, Regensburg, Germany. E-mail: frank-michael.matysik@ chemie.uni-regensburg. de identification. Thirdly, in cases of analytes that show low ionization efficiency in the ESI process a considerable improvement in sensitivity of MS detection can be achieved by the electrochemical conversion of the parent compounds. ${ }^{6}$

EAI is conducted in a special electrochemical injection cell represented schematically in Fig. $1 .^{7}$ The experimental arrangement of a fused silica capillary above a disk electrode forms a thin layer configuration with a fixed capillary-toelectrode distance of $50 \pm 10 \mu \mathrm{m}$. The injection cell contains the sample solution. The injection into the capillary flow system is done hydrodynamically in the absence of the high voltage field used for CE separations. Therefore, the potential of the disk electrode can be controlled without any interference from the high voltage field. After the injection step the capillary inlet is placed in a vial containing the separation buffer and the separation voltage is applied. The separation capillary is directly coupled to ESI-MS detection.

The complete analytical system, consisting of electrochemically assisted injection, capillary electrophoresis and mass spectrometry (EAI-CE-MS), was investigated using a model mixture of four ferrocene derivatives. Ferrocene compounds, first synthesized in the 50s by Kealy and Pauson ${ }^{8}$ and Miller et al. ${ }^{9}$ continue to attract much interest in various fields of chemistry. Ferrocene and corresponding

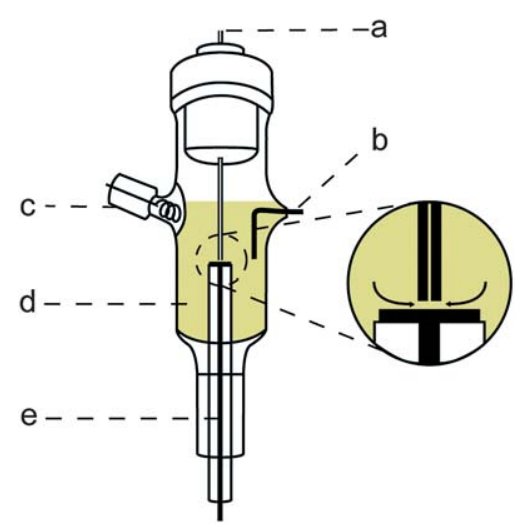

Fig. 1 Schematic representation of the cell configuration for electrochemically assisted injection (EAI): (a) fused silica capillary (ID, $75 \mu \mathrm{m}$ ), (b) counter electrode, (c) Ag quasi-reference electrode, (d) sample solution, and (e) Pt disk electrode $\left(d_{\mathrm{Pt}}=2 \mathrm{~mm}\right)$. 
derivatives are well suited model compounds for EAI-CE-MS studies. Their redox properties enable the generation of positively charged ferrocenium species in a simple one-electron transfer process at moderate potentials around $0.5 \mathrm{~V} v s$. SHE. In addition, ferrocene compounds are readily soluble in acetonitrile which is a very favorable solvent for electrochemistry and capillary electrophoresis. Thus, acetonitrile based non-aqueous capillary electrophoresis (NACE) can be applied. ${ }^{10,11}$ For the studies presented here the electrolyte solution was acetonitrile containing $1 \mathrm{M}$ acetic acid/10 $\mathrm{mM}$ ammonium acetate. This electrolyte system is ideally suited for ESI-MS measurements since all components are easily evaporated.

The model analytes include ferrocene, ferrocene methanol, decamethylferrocene and octamethyl-5,5'-di(2-pyridyl)ferrocene. The chemical structures of these compounds are illustrated in Scheme 1. The compounds decamethylferrocene and octamethyl-5,5'-di(2-pyridyl)ferrocene exhibit special properties in the context of EAI-CEMS. Although both mother compounds are neutral substances, charged species can be formed without the help of EAI. Decamethylferrocene is easily oxidized by dissolved oxygen due to its low redox potential. ${ }^{12}$ The special stability of the decamethylferrocenium ion has been reported by Bashkin and Kinlen. ${ }^{13}$ Octamethyl-5,5'-di(2-pyridyl)ferrocene can be protonated at one pyridyl substituent in the electrolyte medium used. ${ }^{14}$ Scheme 1 shows the mechanisms of charge generation.

Fig. 2 shows two electropherograms for the investigation of a mixture of the above model analytes. The upper electropherogram represents a CE-MS recording without EAI whereas the lower one shows the result in conjunction with EAI. Without EAI only the two cationic species, namely the decamethylferrocenium ion and the protonated octamethyl-5,5'-di(2-pyridyl)ferrocene appear in the CEMS recording. The neutral analytes ferrocene and ferrocene methanol cannot be separated and detected by CE-MS. There are only

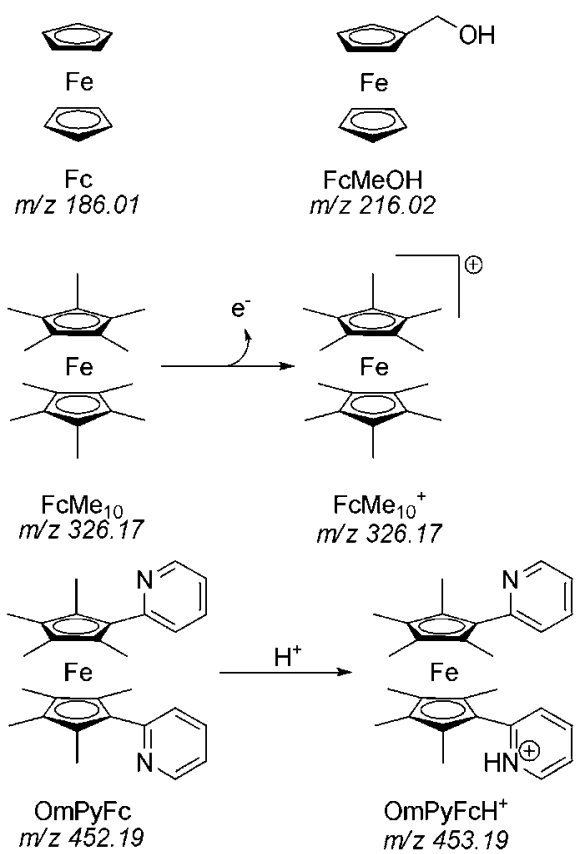

Scheme 1 Model compounds for EIA-CE-MS: ferrocene ( $\mathrm{Fc})$, ferrocene methanol $(\mathrm{FcMeOH})$, decamethylferrocene $\left(\mathrm{FcMe}_{10}\right)$ and octamethyl5,5'-di(2-pyridyl)ferrocene (OmPyFc).
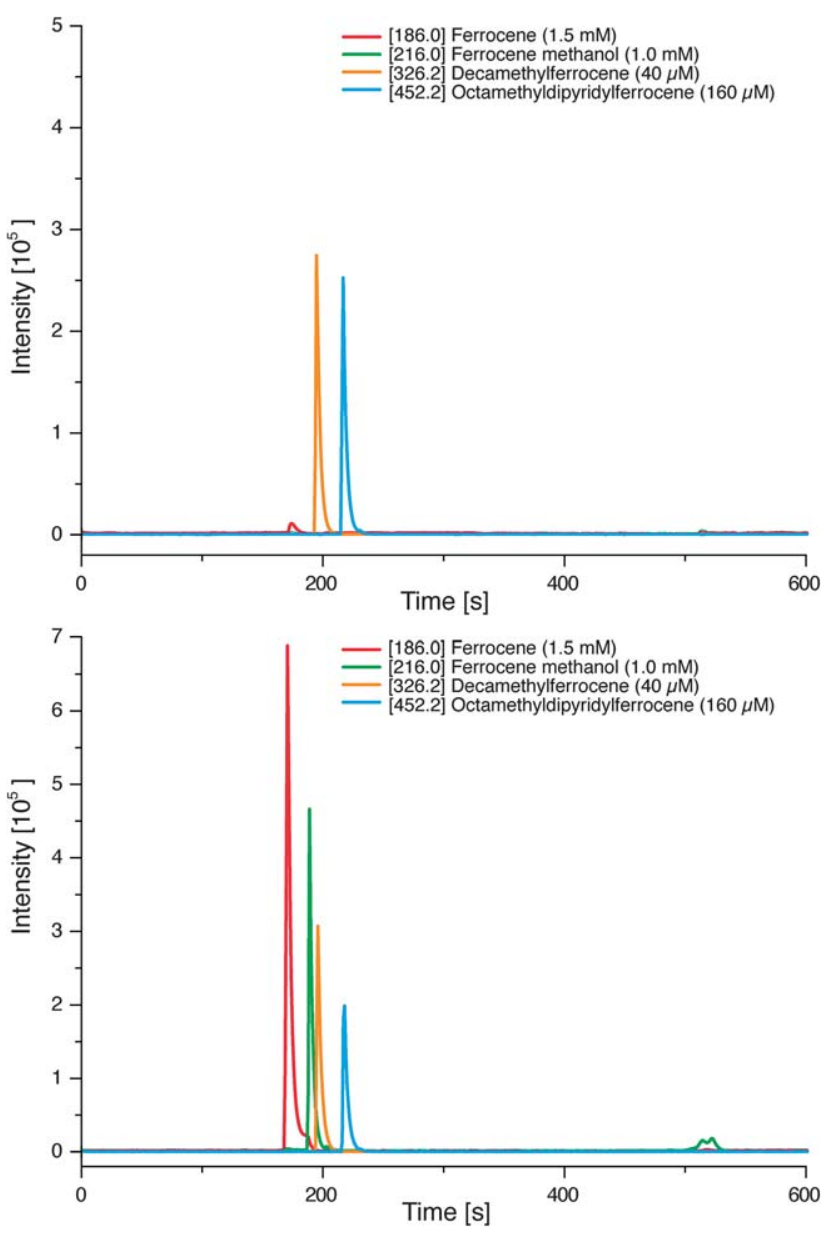

Fig. 2 Electropherograms for CE-MS studies of ferrocene (1.5 mM), ferrocene methanol $(1.0 \mathrm{mM})$, decamethylferrocene $(40 \mu \mathrm{M})$ and octamethyl-5,5'-di(2-pyridyl)ferrocene $(160 \mu \mathrm{M})$ without EAI (top) and with EAI, $E=1 \mathrm{~V}$ (bottom). A separation buffer consisting of acetonitrile/1 M acetic acid/10 mM ammonium acetate was used. The separation was performed with a fused silica capillary $(50 \mu \mathrm{m} \times 70 \mathrm{~cm})$ applying a separation voltage of $30 \mathrm{kV}$.

minor signal traces for the neutral species which are solely transported by the electroosmotic flow (migration time, ca. $520 \mathrm{~s}$ in Fig. 2).

Ferrocene and ferrocene methanol are quite hydrophobic analytes and are not protonated in the ESI source. Oxidation of these ferrocene species associated with the ESI process, as reported by $\mathrm{Xu}$ et al. ${ }^{15}$ has apparently only a minor importance under the present experimental conditions of the sheath-flow CE-ESI interface. If EAI comes into play (lower electropherogram in Fig. 2) the separation and detection of all four ferrocene derivatives can be achieved as a result of the electrochemical generation of cations. The originally uncharged analytes (ferrocene and ferrocene methanol) are converted into corresponding oxidized species which opens the way for their electrophoretic separation and ESI-MS detection. It should be noted, however, that depending on the design of the EAI cell a certain amount of unreacted neutral analyte is injected, i.e., the coulometric efficiency of EAI is usually less than $100 \%$.

The concept of EAI-CE-MS can also be applied to mechanistic studies. As an example the protonation/oxidation behaviour of octamethyl-5,5'-di(2-pyridyl)ferrocene was studied by EAI-CE-MS. Fig. 3 compares the mass spectra corresponding to CE-MS 

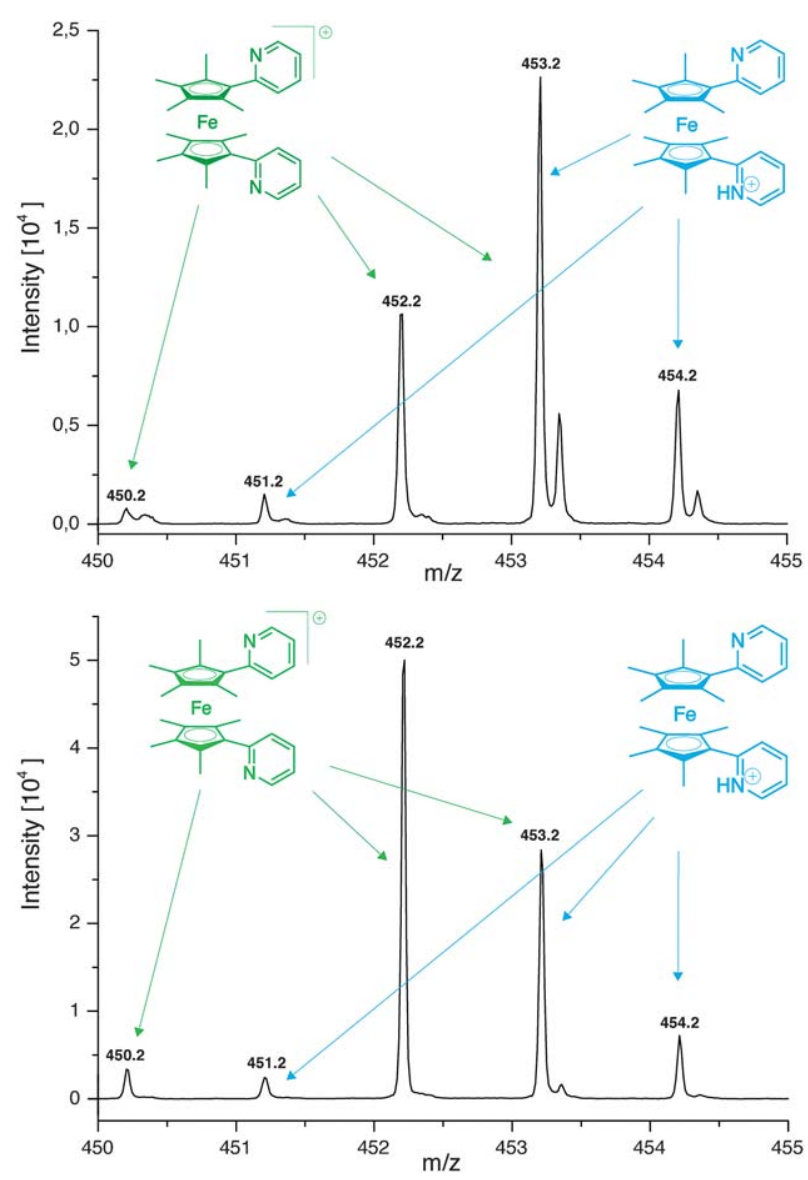

Fig. 3 Mass spectra for CE-MS signals of octamethyl-5,5'-di(2-pyridyl)ferrocene without EAI (top) and with EAI (below). Other experimental conditions are as in Fig. 2.

measurements without and with EAI. Iron containing species exhibit a characteristic isotopic pattern as illustrated in Fig. 3. In general, independent of the use of EAI two cationic species were identified under the present CE-ESI-MS conditions. Without EAI the protonated octamethyl-5,5'-di(2-pyridyl)ferrocene (base peak $m / z=453.2$ with iron in the oxidation state +2 ) is the predominating species. However, the non-protonated but oxidized form of octamethyl-5,5'-di(2-pyridyl)ferrocene (base peak $m / z=452.2$ with iron in the oxidation state +3 ) was also found. This could be attributed to some oxidative reaction in the ESI process. Under conditions of EAI $(E=1 \mathrm{~V})$ the distribution of the two species is drastically changed indicating that the oxidized (deprotonated) form of octamethyl-5, 5'-di(2-pyridyl)ferrocene is predominately generated by means of EAI and migrates in this state through the CE capillary. Obviously, the electrooxidation of octamethyl-5,5'-di(2-pyridyl)ferrocene is associated with a deprotonation. This conclusion is evidenced by the fact that no divalent cationic species could be detected.

The two existing cationic species exhibit only very minor differences regarding their electrophoretic mobility. Consequently, CE separation cannot be realized. However, Fig. 4 illustrates that the MS detection enables to distinguish between both co-eluting species.

Further research in the field of EAI-CE-MS will address the development of miniaturized EAI cells enabling the investigation of

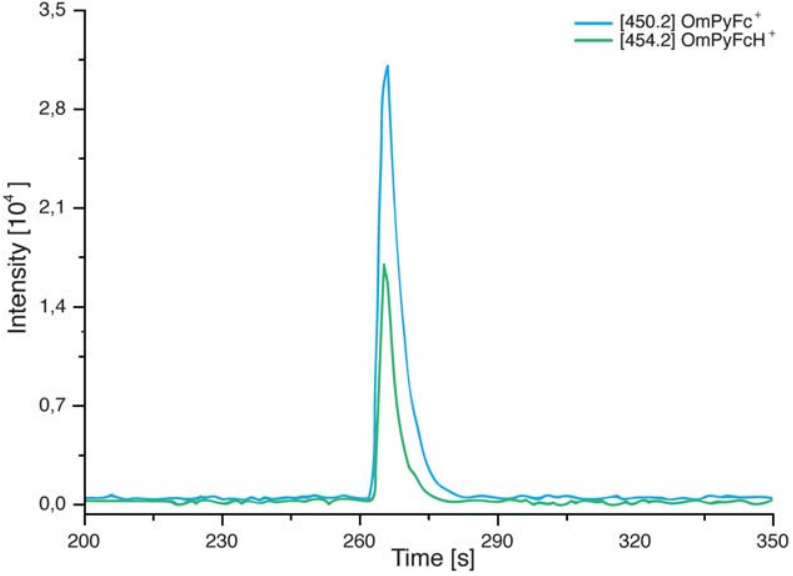

Fig. 4 Electropherogram of octamethyl-5,5'-di(2-pyridyl)ferrocene $(\mathrm{OmPyFc})$ recorded in the frame of an EAI-CE-MS experiment. The two extracted mass traces correspond to the selective isotope signals of the oxidized $\left(\mathrm{OmPyFc}^{+}\right)$and protonated $\left(\mathrm{OmPyFcH}^{+}\right)$species, respectively. Experimental conditions were as in Fig. 2.

very small sample volumes and the use of alternative electrode materials, in particular glassy carbon. In addition, the use of disposable screen-printed electrodes would be a means to overcome potential problems of electrode fouling. Based on these further developments a variety of bioanalytical and pharmaceutical studies in aqueous solution can be envisioned.

\section{Experimental}

All EAI experiments were carried out using the electrochemical injection cell shown in Fig. 1. This cell was used in conjunction with a potentiostat ( $\mu$-Autolab III, Metrohm, Herisau, Switzerland). For the EAI of ferrocene compounds a potential of $1 \mathrm{~V} v s$. Ag quasi-reference electrode was applied.

A micrOTOF-MS (Bruker Daltonik, Bremen, Germany) was coupled with a home-made CE system by means of a coaxial sheathliquid sprayer (Agilent Technologies, Waldbronn, Germany) using isopropanol/water $(50: 50, \mathrm{v} / \mathrm{v})$ containing $0.2 \%$ formic acid as the sheath liquid. Electrical contact at the electrospray needle was established via the sheath liquid delivered at a flow rate of $4 \mu \mathrm{L} \mathrm{min}{ }^{-1}$. Further details concerning this experimental configuration are given in ref. 16 .

The CE system consisted of a high-voltage power supply (Model HCN 7E-35000, F.u.G. Elektronik, Rosenheim-Langenpfunzen, Germany) and a manual sample injection system. ${ }^{17}$ For the CE separations fused silica capillaries $(50 \mu \mathrm{m} \times 70 \mathrm{~cm})$ from Polymicro Technologies (Phoenix, AZ, USA) were used. The separation voltage was $30 \mathrm{kV}$. Sample introduction was carried out by hydrodynamic injection keeping the electrochemical injection cell at an elevated height $(10 \mathrm{~cm})$ relative to the sprayer tip for a period of $10 \mathrm{~s}$. Before and after the injection the capillary inlet was in a buffer vial which was filled with acetonitrile containing $1 \mathrm{M}$ acetic acid/10 mM ammonium acetate.

The ferrocene compounds were purchased from Sigma-Aldrich (Steinheim, Germany); the octamethyl-5,5'-di(2-pyridyl)ferrocene was synthesized and kindly provided by the laboratory of Prof. U. Siemeling (Kassel, Germany). 


\section{Acknowledgements}

This work was supported by the Deutsche Forschungsgemeinschaft (MA 1491/7-1). A sample of octamethyl-5,5'-di(2-pyridyl)ferrocene was kindly provided by Prof. U. Siemeling, Kassel, Germany.

\section{Notes and references}

1 C. P. Palmer and V. T. Remcho, Anal. Bioanal. Chem., 2002, 327, 35.

2 S. Terabe, K. Otsuka, K. Ischikawa, A. Tsuchija and T. Ando, Anal. Chem., 1984, 56, 111.

3 K. L. Rundlett and D. W. Armstrong, Anal. Chem., 1996, 68, 3493.

4 G. W. Somsen, R. Mol and G. J. de Jong, Anal. Bioanal. Chem., 2006, 384, 31 .
5 M. Frommberger, M. Englmann and P. Schmitt-Kopplin, Methods Mol. Biol., 2008, 384, 135.

6 B. Seiwert and U. Karst, Anal. Bioanal. Chem., 2008, 390, 181.

7 F.-M. Matysik, Electrochem. Commun., 2003, 5, 1021.

8 T. J. Kealy and P. L. Pauson, Nature, 1951, 168, 1039.

9 S. A. Miller, J. A. Tebboth and J. F. Tremaine, J. Chem. Soc., 1952, 632.

10 F.-M. Matysik, J. Chromatogr., A, 1998, 802, 349.

11 M.-L. Riekkola, Electrophoresis, 2002, 23, 3865.

12 P. Jutzi, N. Lenze, B. Neumann and H.-G. Stammler, Angew. Chem., 2001, 113, 1469.

13 J. K. Bashkin and P. J. Kinlen, Inorg. Chem., 1990, 29, 4507.

14 B. Neumann, et al., J. Chem. Soc., Dalton Trans., 1997, 4705.

15 X. Xu, S. P. Nolan and R. B. Cole, Anal. Chem., 1994, 66, 119.

16 F.-M. Matysik, C. Neusüß and M. Pelzing, Analyst, 2008, 133, 1764.

17 F.-M. Matysik, J. Chromatogr., A, 1999, 853, 27. 